\title{
The effects of exercise on cardiovascular biomarkers in patients with chronic heart failure
}

\author{
Tariq Ahmad, MD, MPH ${ }^{a, b}$, Mona Fiuzat, PharmD ${ }^{a, b}$, Daniel B. Mark, MD, MPH ${ }^{a, b}$, Ben Neely, \\ MS $^{b}$, Megan Neely, PhD ${ }^{b}$, William E. Kraus, MD ${ }^{a}$, Dalane W. Kitzman, MD $^{c}$, David J. \\ Whellan, MD ${ }^{d}$, Mark Donahue, MD ${ }^{a}$, Faiez Zannad, MD ${ }^{e}$, lleana L. Piña, MD, MPH ${ }^{f}$, Kirkwood \\ Adams, MDg, Christopher M. O'Connor, MD ${ }^{a, b}$, and G. Michael Felker, MD, MHS ${ }^{a}, b$ \\ aDivision of Cardiology, Duke University Medical Center, Durham, NC

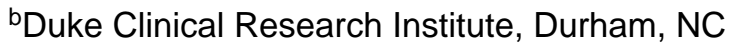 \\ 'Wake Forest University, Winston-Salem, NC \\ dThomas Jefferson University, Philadelphia, PA \\ eNancy University, Nancy, France \\ 'Montefiore Medical Center, Bronx, NY \\ gUniversity of North Carolina, Chapel Hill, NC
}

\section{Abstract}

Background-Exercise training is recommended for chronic heart failure (HF) patients to improve functional status and reduce risk of adverse outcomes. Elevated plasma levels of aminoterminal pro-brain natriuretic peptide (NT-proBNP), high-sensitivity C-reactive protein (hs-CRP), and cardiac troponin $\mathrm{T}$ (cTnT) are associated with increased risk of adverse outcomes in this patient population. Whether exercise training leads to improvements in biomarkers and how such improvements relate to clinical outcomes are unclear.

Methods and Results-Amino-terminal pro-brain natriuretic peptide, hs-CRP, and cTnT levels were assessed at baseline and 3 months in a cohort of 928 subjects from the HF-ACTION study, a randomized clinical trial of exercise training versus usual care in chronic HF patients with reduced left ventricular ejection fraction $(<35 \%)$. Linear and logistic regressions were used to assess 3month biomarker levels as a function of baseline value, treatment assignment (exercise training vs usual care), and volume of exercise. Linear regression and Cox proportional hazard modeling were used to evaluate the relations between changes in biomarker levels and clinical outcomes of interest that included change in peak oxygen consumption (peak $\mathrm{VO}_{2}$ ), hospitalizations, and mortality. Exercise training was not associated with significant changes in levels of NT-proBNP $(P=.10)$, hs-CRP $(P=.80)$, or detectable cTnT levels $(P=.83)$ at 3 months. Controlling for baseline biomarker levels or volume of exercise did not alter these findings. Decreases in plasma concentrations of NT-proBNP, but not hs-CRP or cTnT, were associated with increases in peak $\mathrm{VO}_{2}(P<.001)$ at 3 months and decreased risk of hospitalizations or mortality $(P \leq .04)$, even after adjustment for a comprehensive set of known predictors.

(C) 2013, Mosby, Inc. All rights reserved.

Reprint requests: G. Michael Felker, MD, MHS, Duke Clinical Research Institute, DUMC Box 3850, Durham, NC 27710. michael.felker@duke.edu.

Clinical trial registration: ClinicalTrials.gov no. NCT00047437.

None of the other authors report any conflicts. 
Conclusions-Exercise training did not lead to meaningful changes in biomarkers of myocardial stress, inflammation, or necrosis in patients with chronic HF. Only improvements in NT-proBNP translated to reductions in peak $\mathrm{VO}_{2}$ and reduced risk of clinical events.

Both the 2012 European Society of Cardiology and 2013 American College of Cardiology Foundation/American Heart Association guidelines for diagnosis and management of heart failure (HF) recommend exercise training or regular physical activity for HF patients, to improve functional status and reduce risk of hospitalization (class $1 /$ level of evidence: A). ${ }^{1,2}$ These recommendations were largely based on the definitive trial of exercise in HF-HFACTION — that randomly assigned 2,331 chronic systolic HF patients to either exercise training or usual care for 3 months and showed an $11 \%$ reduction in all-cause mortality, cardiovascular disease mortality, or hospitalizations in the exercise training group. Therefore, although exercise might be beneficial in this patient population, contemporary evaluations to gauge disease stability are quite imprecise; as a result, biomarkers reflecting molecular processes involved in the pathophysiology of HF are increasingly used as clinical tools for disease management. ${ }^{3}$ There is significant evidence in support of using aminoterminal pro-brain natriuretic peptide (NT-proBNP) levels for prognosis (class I/A) and to guide outpatient therapy in $\mathrm{HF}$ (class IIa/B) and emerging evidence in the case of cardiac troponin and high-sensitivity C-reactive protein (hs-CRP) levels. ${ }^{1,4}$

Despite the centrality of exercise as a patient-centered intervention in HF and the increasingly routine use of biomarkers to follow HF patients, the effects of exercise on commonly measured cardiovascular biomarkers have never been assessed in a randomized controlled exercise training trial of HF patients with quantitative measures of exercise performance and cardiovascular fitness. Nonetheless, the positive effects of exercise on cardiovascular biomarkers, particularly on natriuretic peptides levels, have been propagated in the literature, likely due to results from small trials, observational studies, and plausible presupposition. ${ }^{5}$

To definitively examine this hypothesis, we sought to examine the independent effects of exercise training on plasma levels of NT-proBNP, hs-CRP, and cardiac troponin T (cTnT) in a cohort of 928 chronic HF patients who participated in the HF-ACTION trial. We also investigated the relationship between longitudinal improvements in plasma biomarker levels, functional status, and clinical outcomes.

\section{Methods}

\section{Study population}

Details of the design, rationale, and primary results of the HF-ACTION study have been published elsewhere ${ }^{6,7}$ Briefly, HF-ACTION (clinicaltrials.gov: NCT00047437) was a randomized clinical trial evaluating the effect of exercise training versus usual care on longterm morbidity and mortality in 2,331 patients with chronic HF due to left ventricular systolic dysfunction (New York Heart Association [NYHA] classes II-IV, left ventricular ejection fraction $[\mathrm{LVEF}]<35 \%)$.

\section{Exercise testing}

Patients were randomized to either (1) usual HF care or (2) a structured, group-based, supervised exercise program, with a goal of 3 sessions per week for a total of 36 sessions in 3 months. During the supervised training phase, patients performed walking, treadmill, or stationary cycling as their primary training mode. Exercise was initiated at 15 to 30 minutes per session at a heart rate corresponding to $60 \%$ of heart rate reserve (maximal heart rate on cardiopulmonary exercise test minus resting heart rate) and ramped up. Patients in the exercise training group were also provided home exercise equipment, and home exercise 
adherence and amount were formally measured. Patients in the usual care group were not provided with a formal exercise prescription. All patients, regardless of treatment group, received detailed self-management educational materials that included information on medications, fluid management, symptom exacerbation, sodium intake, and amount of activity recommended by the American College of Cardiology and American Heart Association guidelines. ${ }^{8}$ Cardiopulmonary exercise testing and a 6-minute walk test were performed at baseline and at 3 months.

\section{Clinical end points}

The primary end point of HF-ACTION was a composite of all-cause mortality and all-cause hospitalization over a median follow-up of 2.5 years. An independent clinical events committee adjudicated all deaths and first hospitalizations. Additional end points of interest were all-cause mortality, the composite end point of cardiovascular mortality or cardiovascular hospitalization, and the composite end point of cardiovascular mortality or HF hospitalization. HF-ACTION was approved by local institutional review boards, and all enrolled patients provided written informed consent.

\section{Biomarker measurements}

A subset of patients enrolled in the HF-ACTION study agreed to participate in the biomarker substudy and underwent plasma collection at baseline, 3 months, and 12 months. The current analysis was performed on patients with data available on all 3 biomarkers (NTproBNP, hs-CRP, and cTnT) at baseline and 3 months $(\mathrm{n}=928)$. Blood samples were obtained on the same day as exercise testing but were obtained before exercise. Samples were collected via peripheral vein into EDTA containing tubes and then centrifuged immediately and stored at $-70^{\circ} \mathrm{C}$ for subsequent analysis. Amino-terminal pro-brain natriuretic peptide, hs-CRP, and cTnT were measured using enzyme-linked immunosorbent assays (Roche Diagnostics, Indianapolis, IN). ${ }^{9-11}$ All testing was performed at academic core laboratory blinded to clinical data.

\section{Statistical analysis}

Baseline characteristics were described using medians and intraquartile ranges or proportions, according to treatment group (usual case vs exercise training). Differences were compared using Fisher exact and Wilcoxon rank sum tests for continuous variables and $\chi^{2}$ tests for categorical variables. The outcome variables of interest included the clinical outcomes summarized above as well as the changes in peak oxygen consumption (peak $\mathrm{VO}_{2}$ ) from baseline to 3 months. To examine the associations between exercise training and improvements in biomarker levels at 3 months, linear and logistic regression models were fit to assess 3-month levels as a function of baseline level and treatment assignment (exercise training vs usual care). Amino-terminal pro-brain natriuretic peptide and hs-CRP levels were considered as continuous variables and log transformed for analysis because they were not normally distributed. Because cTnT was only detectable in $14 \%$ of subjects, it was modeled as a dichotomous variable, stratified at the lower limit of detection for the assay (high $>0.01$ $\mathrm{ng} / \mathrm{mL}$, low $₫ 0.01 \mathrm{ng} / \mathrm{mL}$ ). To assess the effect of exercise volume, patients in the exercise training arm were grouped according to achieved levels of exercise: low exercise $(<3$ METhours/week), moderate exercise (3-7 MET-hours/week), and high exercise (>7 MET-hours/ week). ${ }^{12}$ The association between volume of exercise and improvements in biomarker levels at 3 months was examined by replacing treatment assignment with categorized exercise volume in the linear model described above. Next, linear and Cox proportional hazards regression models were fit to examine the association between improvement in 3-month biomarker levels and outcomes. Improvements in NT-proBNP and hs-CRP were defined as a numerical decrease in biomarker level from baseline measurement. Improvements in cTnT were defined as categorical changes from high at baseline to low at 3 months. To examine 
the association between improvements in biomarker levels and change in peak $\mathrm{VO}_{2}$, a linear regression model was fit to assess 3-month levels as a function of baseline peak $\mathrm{VO}_{2}$ level and an indicator of biomarker improvement as defined above. To examine the association between improvement in biomarker levels and the remaining outcomes, Cox proportional hazard models landmarked at 3 months were fit that modeled time-to-first event as a function of the baseline biomarker measurement and an indicator for improvement in biomarker level between baseline and 3 months. Adjustments were made using a previously identified set of predictors for each outcome of interest that had been identified in the final adjusted model for the overall HF-ACTION cohort (see online Appendix for final adjusted model for each end point). All analyses were performed with SAS 9.2 (SAS Institute Incorporated, Cary, NC) and R 12.15.1 (R Development Core Team; R Foundation for Statistical Computing, Vienna, Austria). $P \leq .05$ was considered statistically significant for all analyses. The authors had full access to and take full responsibility for the integrity of the data.

Dr Ahmad received support from the Daland Fellowship in Clinical Investigation. The HFACTION study was funded by grants from the National Heart, Lung, and Blood Institute. The biomarkers assays were funded by a grant from Roche Diagnostics. No other extramural funding was used to support this work. The authors are solely responsible for the design and conduct of this study, all study analyses, the drafting and editing of the manuscript, and its final contents.

\section{Results \\ Baseline characteristics}

Baseline and 3-month plasma levels of NT-proBNP, hs-CRP, and cTnT were available for 928 patients, who were broadly similar to the HF-ACTION cohort as a whole $(\mathrm{n}=2,331$, Supplementary Table I). Patient characteristics of this cohort, as a whole and according to randomization group, are shown in Table I. Median age of the study cohort was 59 years; of these, $63 \%$ were white, and $71 \%$ were male. Most patients were NYHA class II (67\%) or NYHA class III (32\%) at study onset, and median LVEF was 25\%. Enrolled patients had a high utilization of evidence-based therapies for systolic $\mathrm{HF}$, with $94 \%$ receiving $\beta$-blockers and $95 \%$ receiving either angiotensin-converting enzyme inhibitors or angiotensin receptor blockers. Median NT-proBNP levels were $819 \mathrm{pg} / \mathrm{mL}$; median hs-CRP levels were $3.3 \mathrm{mg} /$ $\mathrm{dL}$, and $13.9 \%$ of subjects had cTnT $>0.01 \mu \mathrm{g} / \mathrm{mL}$. There were no statistically significant differences between the usual care and exercise training groups for any of the variables at baseline.

\section{Changes in biomarkers with exercise training}

There were no statistically significant differences in NT-proBNP (779 vs $694, P=.59$ ), hsCRP (3.4 vs 3.2, $P=.34)$, or the proportion of subjects with cTnT elevation $(14.4 \%$ vs $14.7 \%, P=.91)$ at 3 months between the exercise training and usual care groups. Exercise training did not influence biomarker levels at 3 months, even after controlling for baseline levels of NT-proBNP $(P=.10)$ or hs-CRP $(P=.80)$. Similarly, there was no association between exercise training and control groups with regard to the prevalence of elevated cTnT levels at 3 months $(P=.83)$. Figure $1 \mathrm{~A}$ and $\mathrm{B}$ shows scatter plots of baseline and 3-month measurements for both NT-proBNP and hs-CRP according to treatment group. Figure 1C shows bar graphs of the number of patients in each category of baseline and 3-month cTnT status. 


\section{Volume of exercise and biomarker changes}

Patients were grouped according to achieved level of exercise at 3 months in the exercise training group: low exercise ( $<3$ MET-hours/week), moderate exercise (3-7 MET-hours/ week), and high exercise (>7 MET-hours/week). When compared with patients in the low exercise group, those in the moderate and high exercise group had decreased risk of allcause mortality and hospitalizations (hazard ratio [HR] 0.58, 95\% CI [0.43-0.78] and HR $0.55,95 \%$ CI [0.35-0.860], respectively). Although levels of both NT-proBNP and hs-CRP levels were progressively lower among patients who were able to achieve higher levels of exercise, the differences between baseline and 3-month levels of either biomarker within each exercise category were not significantly different (Figure 2). We were unable to test this hypothesis in the case of cTnT due to the small number of patients who experienced a change from high to low across exercise volume groups.

\section{Changes in biomarker levels, exercise capacity, and long-term outcomes}

Next, we examined the relationships between improvements in biomarker levels, changes in maximal exercise capacity (peak $\mathrm{VO}_{2}$ ), and clinical outcomes. For patients in whom NTproBNP levels decreased at 3 months, there was a concomitant increase in peak $\mathrm{VO}_{2}(P \leq$. 001) (Figure 3A). Improvements in hs-CRP or cTnT were not associated with increases in peak $\mathrm{VO}_{2}(P=.08$ : Figure $3 \mathrm{~B}, P=.78$, Figure $3 \mathrm{C})$.

Improved NT-proBNP levels at 3 months were associated with reduced risk of all 3 composite clinical outcomes: (1) all-cause mortality or all-cause hospitalization, (2) allcause mortality, and (3) cardiovascular mortality or cardiovascular hospitalization $(P \leq .04$ all) (Table II). This decrease in risk persisted even after controlling for a comprehensive set of previously identified predictors for each outcome from the overall HF-ACTION cohort (Supplementary Table II). ${ }^{13}$ Improvements in cTnT were associated with decreased risk of all-cause mortality $(P=.03)$ that did not persist in the fully adjusted analysis. There were no associations between reductions in hs-CRP and clinical outcomes.

\section{Discussion}

Findings from this study challenge the hypothesis that exercise training improves commonly evaluated cardiovascular biomarkers in patients with chronic HF. We found that plasma levels of NT-proBNP, hs-CRP, or cTnT did not significantly improve at 3 months despite a structured exercise training program, even after accounting for baseline biomarker levels. Although patients who tended to exercise more had lower levels of NT-proBNP and hs-CRP and decreased risk of adverse clinic outcomes, volume of exercise did not influence serial levels. Only decreases in NT-proBNP translated to improvements in functional capacity and reductions in risk of clinical outcomes.

We believe that these data have important clinical implications as biomarker-guided therapy becomes more routine in the management of chronic HF, and effects of key therapeutic interventions on serial biomarker levels require clarification. Although exercise training has a class 1 recommendation by both the 2012 European Society of Cardiology and 2013 American College of Cardiology/American Heart Association guidelines for treatment of chronic HF, its effects on serial levels of commonly measured cardiovascular biomarkers are unclear. ${ }^{1,2}$ This study, an analysis of the definitive trial of exercise training in HF that led to the recommendations above, is the first to have standardized ascertainments of detailed exercise data and serial biomarker measurements on HF patients randomized to exercise interventions versus standard of care and found no meaningful effect of exercise on serial levels. 
The most immediate clinical implications of our results relate to the use of exercise to reduce natriuretic peptide levels in patients with chronic HF, a strategy that has been perpetuated in the literature as accepted wisdom. ${ }^{1,5}$ In terms of the individual patients, our findings would suggest that any changes in NT-proBNP levels with exercise training would be minimal and the alternative therapies might be more efficacious for this purpose. Although our negative results in regard to the independent effects of exercise on NTproBNP are at odds with most published studies, previous randomized studies examining this question were small (largest $\mathrm{n}=71$ patients) and varied significantly in design, methodology, and patient characteristics. ${ }^{14}$ A recently published meta-analysis of 10 studies that included 565 patients found exercise to decrease natriuretic peptide levels; however, understanding the results needs cautious interpretation of the data as well as confirmation within a randomized trial, which the current study provides using almost twice as many participants $(\mathrm{n}=928) .{ }^{15,16}$ Lastly, an observational study of 2,933 patients with HF looked at the effects of physical activity on NT-proBNP levels, finding evidence of an inverse relationship between NT-proBNP levels and amount of self-reported physical activity. ${ }^{17,18}$ Our results confirm the negative correlation between volume of exercise and biomarker levels, but not an association between exercise training and improvements in biomarker levels. This implies that the suggested causal relationship between activity and lower NTproBNP levels may have resulted from false-positive associations, a common drawback of observational studies. ${ }^{17}$

Although inflammation and myonecrosis play a central role in the pathogenesis of chronic $\mathrm{HF}$, only a handful of studies have reported on changes in hs-CRP and cTnT with exercise in patients. These studies have not included patients with established HF; to our knowledge, this is the first look at the effects of exercising training on serial hs-CRP and cTnT levels in this patient population. ${ }^{19-22}$ Our results suggest that serial measurements of these biomarkers may not be reliable surrogates for any health benefits of physical activity in this patient population.

Our findings that serial improvements in NT-proBNP lead to reduced risk of clinical outcomes have been previously described in the literature. ${ }^{23,24}$ The fact that our results are consistent with previous findings adds credence to the notion that our null results in regard to NT-proBNP changes with exercise training may be accurate. We also report on a lack of association between reductions in hs-CRP and cTnT and changes in peak $\mathrm{VO}_{2}$ or lowered risk of clinical outcomes. The incremental clinical value of serial hs-CRP measurements in HF patients has never been well studied, and these findings will require future confirmation; however, they reflect results of a recent study showing no predictive value of serial hs-CRP levels in high-risk patients without HF. ${ }^{25,26}$ Lastly, our negative findings in regard to serial cTnT levels run counter to some previous studies. ${ }^{27,28}$ However, results of studies examining this question have varied tremendously, depending on the patient population studied, the troponin assay used, or the study design. ${ }^{29}$ There is currently no clear consensus on the use of cTnT measurements in chronic HF, and the results presented here raise the need for a closer examination of the prognostic implications of serial troponin measurements in HF. ${ }^{29,30}$

There are potential limitations to the present study. The population for this study was derived from the HF-ACTION trial and is susceptible to the limitations inherent in clinical trial populations. Biomarker measurements were made after 3 months of structured exercise training, and there is a possibility that changes in levels might have been noted after a longer exercise period. Our study population consisted only of patients with impaired ejection fraction (LVEF $<35 \%$ ), so our results cannot be extrapolated to the population of patients with $\mathrm{HF}$ and preserved ejection fraction. Our population consisted of ambulatory patients with NYHA class II and III HF, and these findings may not be generalizable to patients with 
less or more severe illness. We used a commonly used platform for measurement of cTnT, rather than the newer high sensitivity assays used in more recent studies of troponin elevation in $\mathrm{HF}^{30}$

In conclusion, a 3-month structured exercise training program did not lead to improvements in plasma concentrations of NT-proBNP, hs-CRP, and cTnT, compared with usual care, in a cohort of stable chronic systolic HF patients. Baseline biomarker levels or volume of exercise did not alter these findings. Serial improvements in NT-proBNP, but not hs-CRP or cTnT levels, were associated with increases in peak $\mathrm{VO}_{2}$ levels and decreased risk of adverse clinical outcomes.

\section{Supplementary Material}

Refer to Web version on PubMed Central for supplementary material.

\section{Acknowledgments}

Disclosures: Drs Felker, Fiuzat, and O'Connor have received research funding from BG Medicine, Critical Diagnostics, and Roche Diagnostics. Drs Felker and O'Connor have served as consultants for Roche Diagnostics. Dr Zannad has received research funding from BG Medicine and Roche Diagnostics and served as a consultant for BG Medicine. Dr Kitzman serves as a consultant for Relypsa, Inc.

\section{References}

1. Yancy CW, Jessup M, Bozkurt B, et al. 2013 ACCF/AHA guideline for the management of heart failure: a report of the American College Of Cardiology Foundation/American Heart Association Task Force on Practice Guidelines. J Am Coll Cardiol. 2013; 62(16):e147-239. [PubMed: 23747642]

2. McMurray JJ, Adamopoulos S, Anker SD, et al. Guidelines ESCCfP ESC guidelines for the diagnosis and treatment of acute and chronic heart failure 2012: The Task Force for the Diagnosis and Treatment of Acute and Chronic Heart Failure 2012 of the European Society of Cardiology Developed in collaboration with the Heart Failure Association (HFA) of the ESC. Eur Heart J. 2012; 33:1787-847. [PubMed: 22611136]

3. Ahmad T, Fiuzat M, Felker GM, et al. Novel biomarkers in chronic heart failure. Nat Rev Cardiol. 2012; 9:347-59. [PubMed: 22450126]

4. Braunwald E. Heart failure. JACC: Heart Fail. 2013; 1:1-20.

5. Januzzi JL, Troughton R. Are serial bnp measurements useful in heart failure management? Serial natriuretic peptide measurements are useful in heart failure management. Circulation. 2013; 127:500-7. discussion 508. [PubMed: 23357662]

6. O'Connor CM, Whellan DJ, Lee KL, et al. Efficacy and safety of exercise training in patients with chronic heart failure: HF-ACTION randomized controlled trial. JAMA. 2009; 301:1439-50. [PubMed: 19351941]

7. Whellan DJ, O'Connor CM, Lee KL, et al. Heart failure and a controlled trial investigating outcomes of exercise training (HF-ACTION): design and rationale. Am Heart J. 2007; 153:201-11. [PubMed: 17239677]

8. Hunt SA, Abraham WT, Chin MH, et al. ACC/AHA 2005 Guideline Update for the Diagnosis and Management of Chronic Heart Failure in the Adult: a report of the American College of Cardiology/ American Heart Association Task Force on Practice Guidelines (Writing Committee to Update the 2001 Guidelines for the Evaluation and Management of Heart Failure): developed in collaboration with the American College of Chest Physicians and the International Society for Heart and Lung Transplantation: endorsed by the Heart Rhythm Society. Circulation. 2005; 112:e154-235. [PubMed: 16160202]

9. Mueller T, Gegenhuber A, Poelz W, et al. Head-to-head comparison of the diagnostic utility of BNP and NT-proBNP in symptomatic and asymptomatic structural heart disease. Clin Chim Acta. 2004; 341:41-8. [PubMed: 14967157] 
10. Ridker PM, Cushman M, Stampfer MJ, et al. Plasma concentration of C-reactive protein and risk of developing peripheral vascular disease. Circulation. 1998; 97:425-8. [PubMed: 9490235]

11. Lindahl B, Diderholm E, Lagerqvist B, et al. Mechanisms behind the prognostic value of troponin $\mathrm{T}$ in unstable coronary artery disease: a FRISC II substudy. J Am Coll Cardiol. 2001; 38:979-86. [PubMed: 11583868]

12. Keteyian SJ, Leifer ES, Houston-Miller N, et al. Relation between volume of exercise and clinical outcomes in patients with heart failure. J Am Coll Cardiol. 2012; 60:1899-905. [PubMed: 23062530]

13. O'Connor CM, Whellan DJ, Wojdyla D, et al. Factors related to morbidity and mortality in patients with chronic heart failure with systolic dysfunction: the HF-ACTION predictive risk score model. Circ Heart Fail. 2012; 5:63-71. [PubMed: 22114101]

14. Smart NA, Steele M. Systematic review of the effect of aerobic and resistance exercise training on systemic brain natriuretic peptide (BNP) and N-terminal BNP expression in heart failure patients. Int J Cardiol. 2010; 140:260-5. [PubMed: 19664831]

15. Smart NA, Meyer T, Butterfield JA, et al. Individual patient meta-analysis of exercise training effects on systemic brain natriuretic peptide expression in heart failure. Eur J Prev Cardiol. 2012; 19:428-35. [PubMed: 21543459]

16. Thompson SG, Pocock SJ. Can meta-analyses be trusted? Lancet. 1991; 338:1127-30. [PubMed: 1682553]

17. deFilippi CR, de Lemos JA, Tkaczuk AT, et al. Physical activity, change in biomarkers of myocardial stress and injury, and subsequent heart failure risk in older adults. J Am Coll Cardiol. 2012; 60:2539-47. [PubMed: 23158528]

18. O'Connor CM, Ahmad T. Can we prevent heart failure with exercise? J Am Coll Cardiol. 2012; 60:2548-9. [PubMed: 23158537]

19. Mora S, Lee IM, Buring JE, et al. Association of physical activity and body mass index with novel and traditional cardiovascular biomarkers in women. JAMA. 2006; 295:1412-9. [PubMed: 16551713]

20. Huffman KM, Samsa GP, Slentz CA, et al. Response of high-sensitivity C-reactive protein to exercise training in an at-risk population. Am Heart J. 2006; 152:793-800. [PubMed: 16996860]

21. Kasapis C, Thompson PD. The effects of physical activity on serum C-reactive protein and inflammatory markers: a systematic review. J Am Coll Cardiol. 2005; 45:1563-9. [PubMed: 15893167]

22. Shave R, Baggish A, George K, et al. Exercise-induced cardiac troponin elevation: evidence, mechanisms, and implications. J Am Coll Cardiol. 2010; 56:169-76. [PubMed: 20620736]

23. Latini R, Masson S, Wong M, et al. Incremental prognostic value of changes in B-type natriuretic peptide in heart failure. Am J Med. 2006; 119:70e23-30. [PubMed: 16431191]

24. Januzzi JL Jr, Maisel AS, Silver M, et al. Natriuretic peptide testing for predicting adverse events following heart failure hospitalization. Congest Heart Fail. 2012; 18(suppl 1):S9-S13. [PubMed: 22891803]

25. Araujo JP, Lourenco P, Azevedo A, et al. Prognostic value of high-sensitivity C-reactive protein in heart failure: a systematic review. J Card Fail. 2009; 15:256-66. [PubMed: 19327628]

26. Blumenthal RS, Ndumele CE, Martin SS. Ask not what crp can do for you. J Am Coll Cardiol. 2013; 62:730-1. [PubMed: 23948515]

27. O'Connor CM, Fiuzat M, Lombardi C, et al. Impact of serial troponin release on outcomes in patients with acute heart failure: analysis from the protect pilot study. Circ Heart Fail. 2011; 4:724-32. [PubMed: 21900185]

28. Felker GM, Hasselblad V, Tang WH, et al. Troponin I in acute decompensated heart failure: insights from the ASCEND-HF study. Eur J Heart Fail. 2012; 14:1257-64. [PubMed: 22764184]

29. Kociol RD, Pang PS, Gheorghiade M, et al. Troponin elevation in heart failure prevalence, mechanisms, and clinical implications. J Am Coll Cardio. 2010; 56(14):1071-8.

30. Januzzi JL Jr, Filippatos G, Nieminen M, et al. Troponin elevation in patients with heart failure: on behalf of the third Universal Definition of Myocardial Infarction Global Task Force: Heart Failure Section. Eur Heart J. 2012; 33:2265-71. [PubMed: 22745356] 


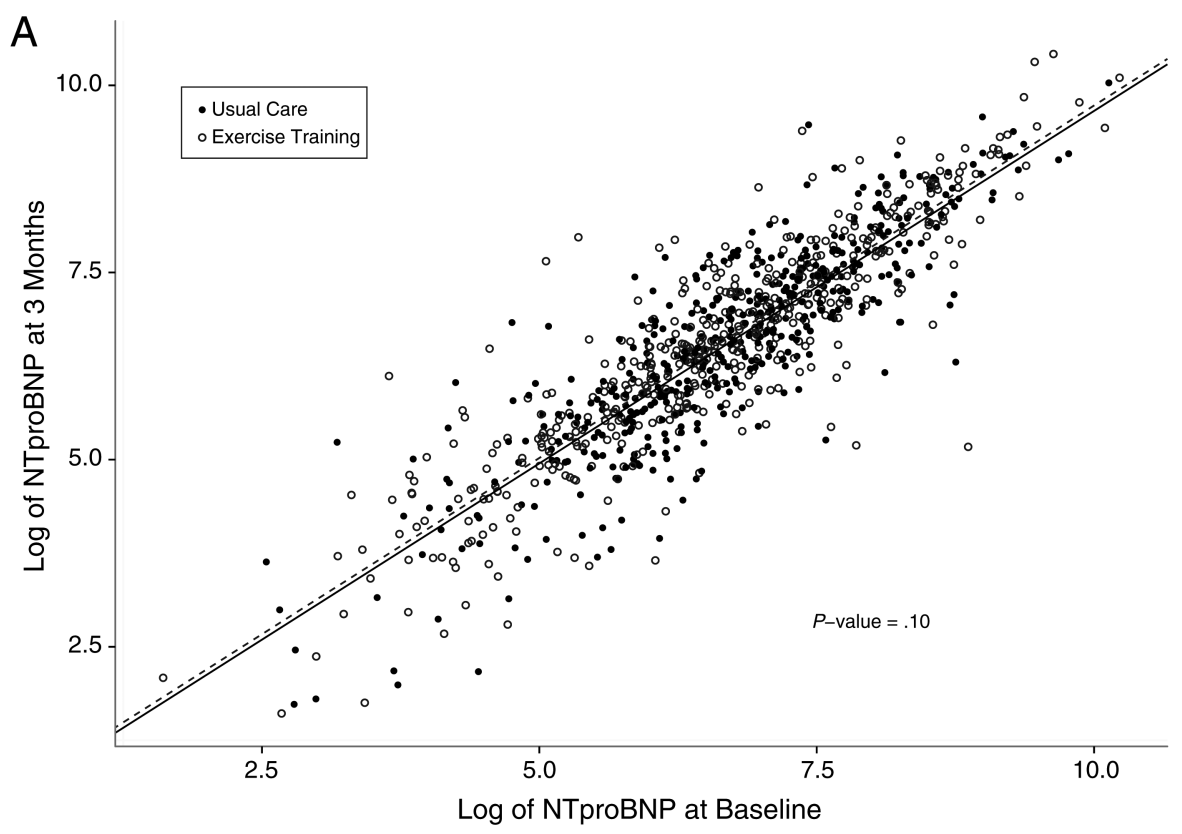

B

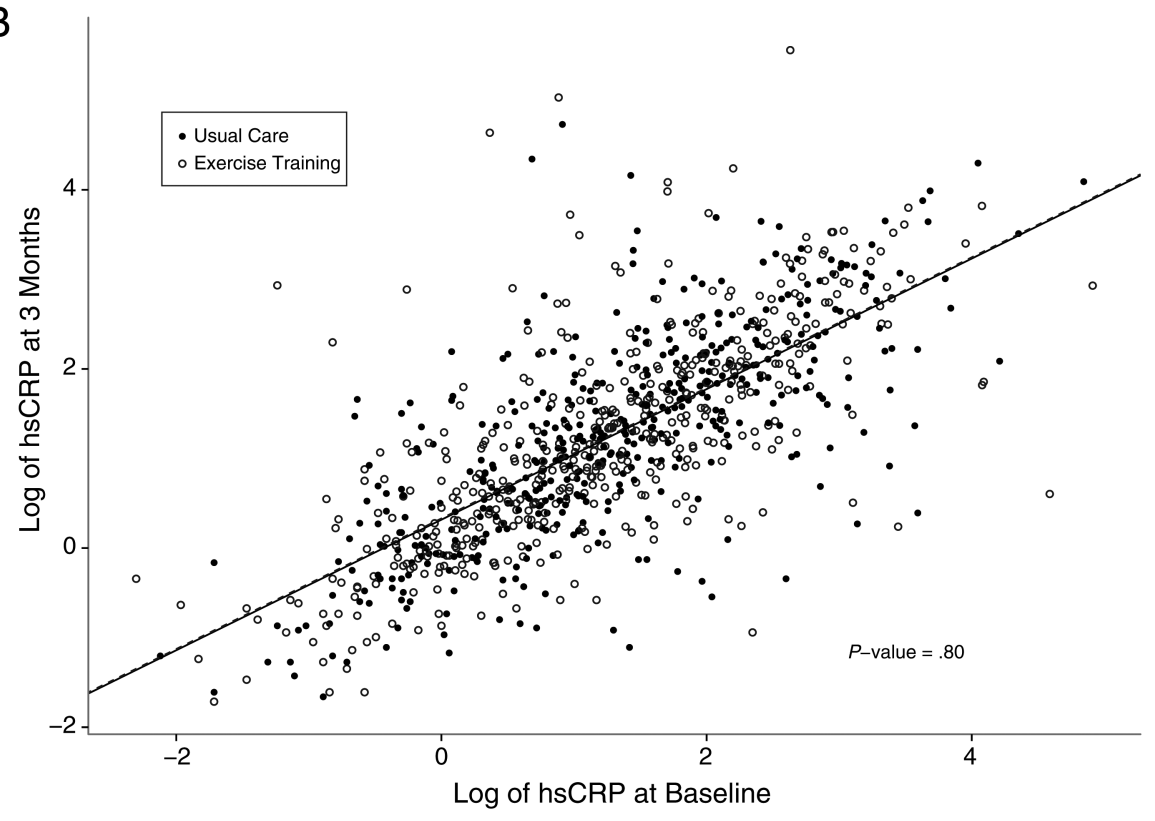

Am Heart J. Author manuscript; available in PMC 2015 February 01. 


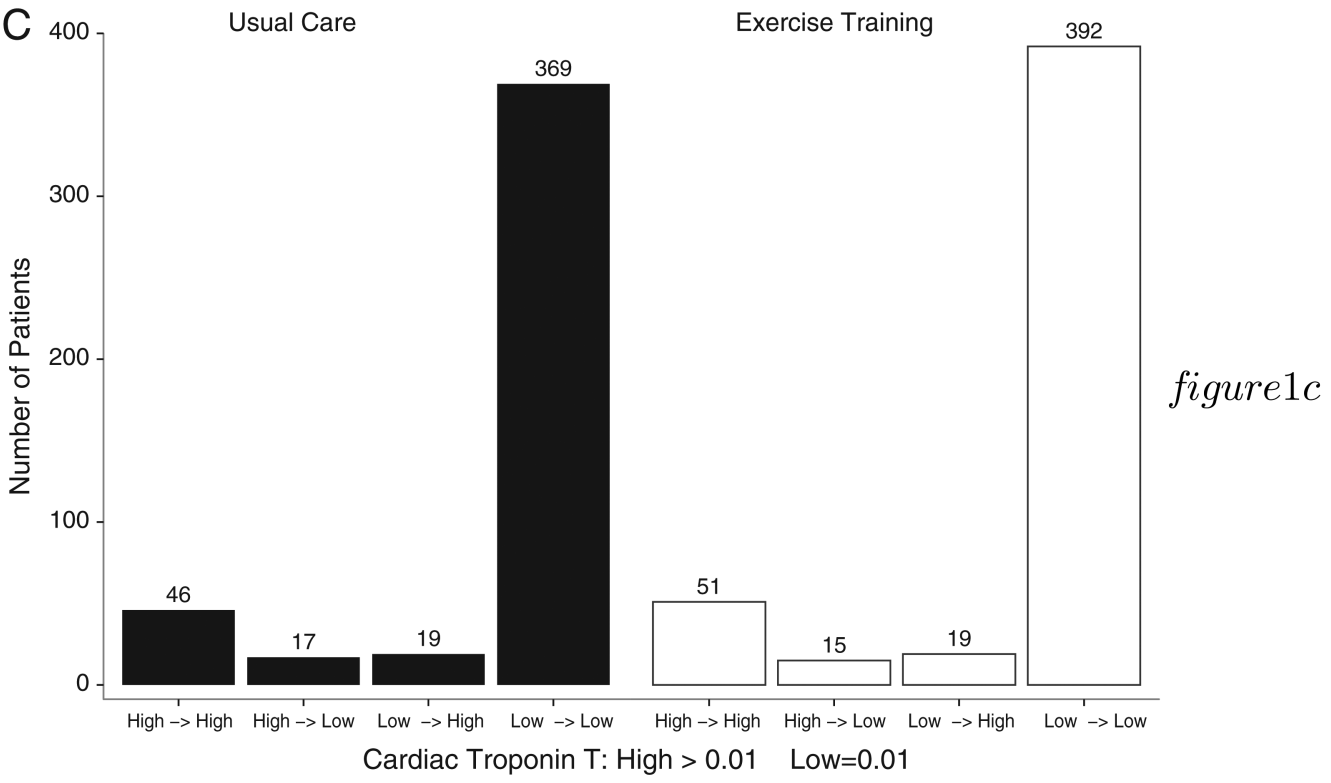

Figure 1.

Baseline and 3-month biomarker levels according to treatment group. Scatter plots depicting the baseline (x-axis) and 3-month (y-axis) measurements of log-transformed NT-proBNP (A) and hs-CRP (B), according to treatment assignment. C, Frequency distributions of baseline (left) and 3-month (right) cTnT, according to treatment assignment. 

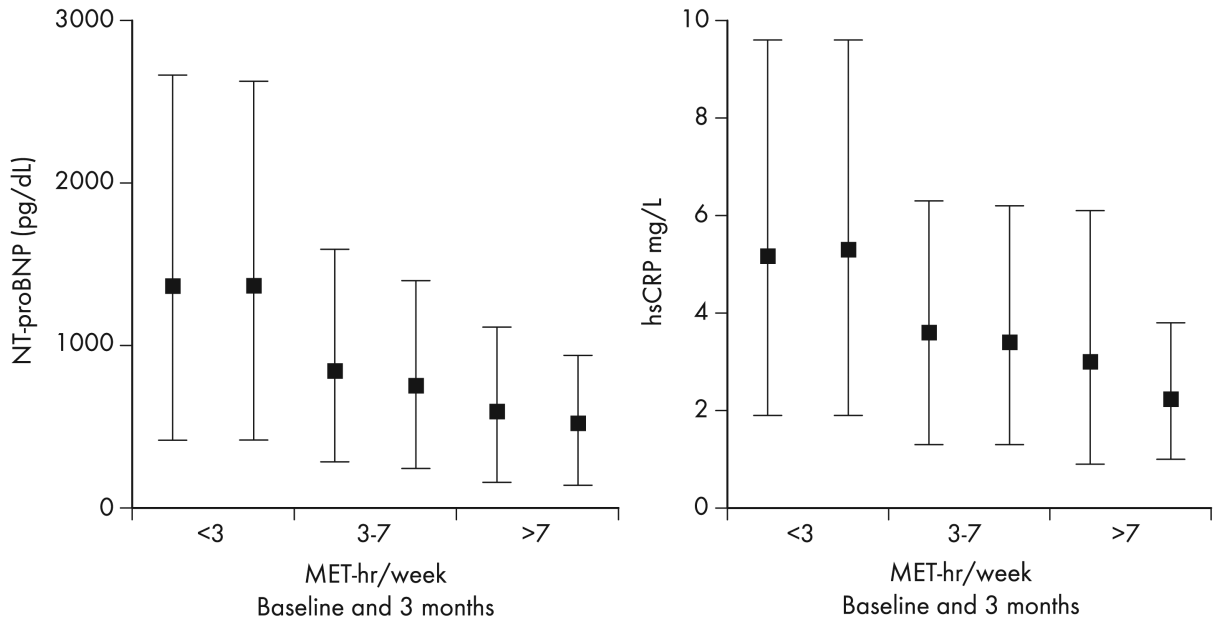

Figure 2.

Baseline and 3-month biomarker levels according to amount of exercise. Median and intraquartile ranges of baseline and 3-month values for NT-proBNP and hs-CRP, according to amount of exercise. $P$ values indicate whether levels between baseline and 3 months are statistically different. 

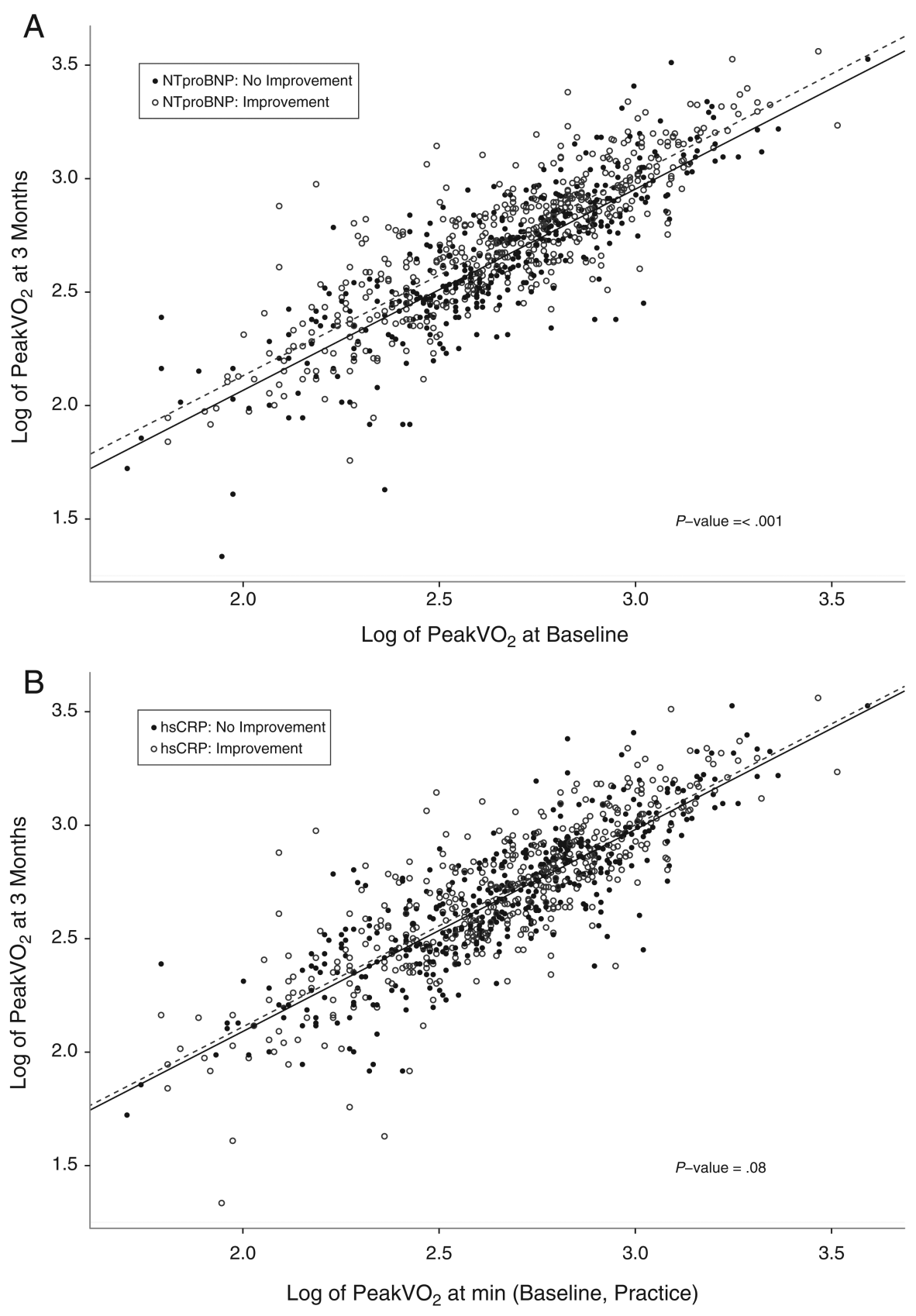

Am Heart J. Author manuscript; available in PMC 2015 February 01. 


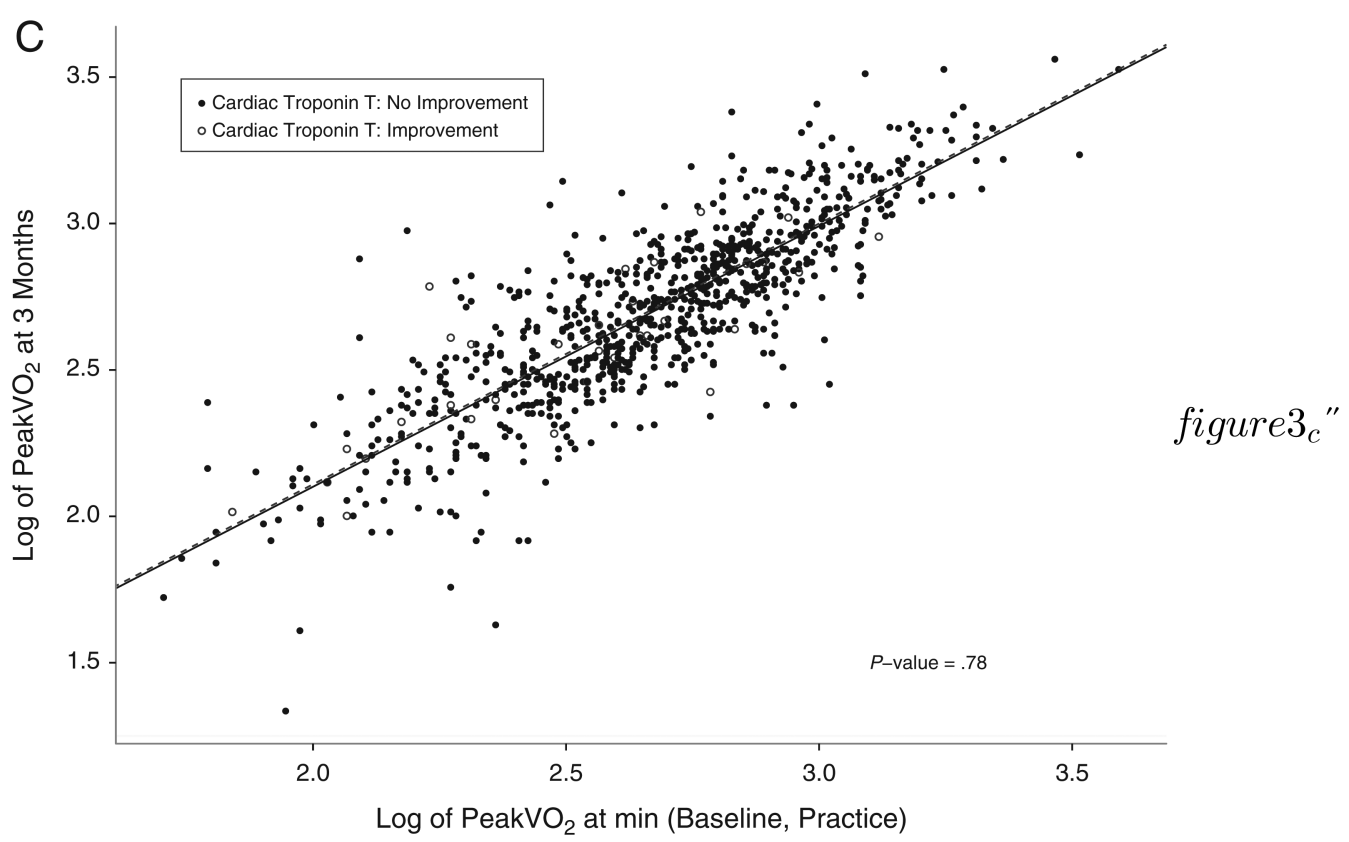

Figure 3.

Change in baseline and 3-month peak $\mathrm{VO}_{2}$ according to biomarker improvement. Scatter plots depicting the baseline (x-axis) and 3 month (y-axis) measurements of log-transformed peak $\mathrm{VO}_{2}$ according to levels of NT-proBNP (A), hs-CRP (B), and cTnT $(\mathbf{C})$, grouped according to biomarker improvement versus no improvement at 3-month follow-up. 
Table I

\section{Baseline characteristics of study participants}

\begin{tabular}{|c|c|c|c|c|}
\hline & Overall population $(\mathrm{n}=928)$ & Usual care $(n=451)$ & Exercise training $(n=477)$ & $P$ \\
\hline Age, y & $59.0(50.9,68.0)$ & $59.0(50.7,68.0)$ & $59.0(51.2,67.9)$ & .919 \\
\hline Sex, \% woman & $272 / 928(29.3 \%)$ & $120 / 451(26.6 \%)$ & $152 / 477(31.9 \%)$ & .079 \\
\hline Race (\% white) & $573 / 912(62.8 \%)$ & $286 / 446(64.1 \%)$ & $287 / 466(61.6 \%)$ & .354 \\
\hline History of diabetes & $303 / 928(32.7 \%)$ & $145 / 451(32.2 \%)$ & $158 / 477(33.1 \%)$ & .752 \\
\hline History of MI & $380 / 928(40.9 \%)$ & $191 / 451(42.4 \%)$ & $189 / 477(39.6 \%)$ & .398 \\
\hline History of hypertension & $568 / 922(61.6 \%)$ & $261 / 449(58.1 \%)$ & $307 / 473(64.9 \%)$ & .034 \\
\hline Current smoker & $145 / 924(15.7 \%)$ & $78 / 449(17.4 \%)$ & $67 / 475(14.1 \%)$ & .344 \\
\hline \multicolumn{5}{|l|}{ NYHA class } \\
\hline II & $619 / 928(66.7 \%)$ & $306 / 451(67.8 \%)$ & $313 / 477(65.6 \%)$ & \\
\hline III & $301 / 928(32.4 \%)$ & $143 / 451(31.7 \%)$ & $158 / 477(33.1 \%)$ & .382 \\
\hline IV & $8 / 928(0.9 \%)$ & $2 / 451(0.4 \%)$ & $6 / 477(1.3 \%)$ & \\
\hline \multicolumn{5}{|l|}{ Resting ECG conduction } \\
\hline Normal & $376 / 914(41.1 \%)$ & $184 / 443(41.5 \%)$ & $192 / 471(40.8 \%)$ & \\
\hline LBBB & $161 / 914(17.6 \%)$ & $78 / 443(17.6 \%)$ & $83 / 471(17.6 \%)$ & .975 \\
\hline RBBB & $35 / 914(3.8 \%)$ & $15 / 443(3.4 \%)$ & $20 / 471(4.2 \%)$ & \\
\hline IVCD & $123 / 914(13.5 \%)$ & $60 / 443(13.5 \%)$ & $63 / 471(13.4 \%)$ & \\
\hline Paced & $219 / 914(24.0 \%)$ & $106 / 443(23.9 \%)$ & $113 / 471(24.0 \%)$ & \\
\hline Creatinine, $\mathrm{mg} / \mathrm{dL}$ & $1.2(1.0,1.5)$ & $1.2(1.0,1.5)$ & $1.2(1.0,1.5)$ & .835 \\
\hline Blood urea nitrogen, $\mathrm{mg} / \mathrm{dL}$ & $21.0(15.0,28.0)$ & $21.0(15.0,29.0)$ & $20.5(15.0,27.0)$ & .911 \\
\hline Hemoglobin level, g/dL & $13.3(12.3,14.5)$ & $13.4(12.3,14.6)$ & $13.3(12.1,14.5)$ & .252 \\
\hline NT-proBNP, pg/mL (B) & $818.8(346.0,1818)$ & $853.2(374.6,1829)$ & $762.3(310.5,1809)$ & .221 \\
\hline NT-proBNP, pg/mL (3 M) & $726.1(281.6,1753)$ & $778.5(296.7,1825)$ & $693.7(276.1,1725)$ & .593 \\
\hline hs-CRP, mg/dL (baseline) & $3.3(1.5,7.8)$ & $3.4(1.6,7.9)$ & $3.2(1.4,7.5)$ & .321 \\
\hline hs-CRP, mg/dL (3 M) & $3.3(1.5,7.6)$ & $3.6(1.6,7.8)$ & $3.1(1.4,7.4)$ & .348 \\
\hline Troponin $\mathrm{T}>0.01 \mu \mathrm{g} / \mathrm{mL}$ (B) & $129 / 928(13.9 \%)$ & $63 / 451(14.0 \%)$ & $66 / 477(13.8 \%)$ & .954 \\
\hline Troponin $\mathrm{T}>0.01 \mu \mathrm{g} / \mathrm{mL}(3 \mathrm{M})$ & $135 / 928(14.5 \%)$ & $65 / 451(14.4 \%)$ & $70 / 477(14.7 \%)$ & .910 \\
\hline LVEF, \% & $24.6(20.0,30.2)$ & $24.7(20.0,30.5)$ & $24.5(20.0,30.1)$ & .606 \\
\hline Severe or moderate MR & $105 / 928(11.3 \%)$ & $52 / 451(11.5 \%)$ & $53 / 477(11.1 \%)$ & .840 \\
\hline Exercise duration, min & $10.0(7.2,12.0)$ & $10.0(7.4,12.3)$ & $9.8(7.0,12.0)$ & .302 \\
\hline Peak $\mathrm{VO}_{2}$ in $\mathrm{mL} / \mathrm{kg} / \mathrm{min}$ & $14.5(11.8,17.5)$ & $14.7(12.0,17.7)$ & $14.4(11.4,17.4)$ & .183 \\
\hline 6-min walk distance, $\mathrm{m}$ & $376.4(304.8,433.7)$ & $374.3(312.1,428.2)$ & $378.1(297.2,441.6)$ & .930 \\
\hline$\beta$-Blocker & $869 / 928(93.6 \%)$ & $428 / 451(94.9 \%)$ & $441 / 477(92.5 \%)$ & .127 \\
\hline $\mathrm{ACE}$ or $\mathrm{ARB}$ & $881 / 928(94.9 \%)$ & $428 / 451(94.9 \%)$ & $453 / 477(95.0 \%)$ & .962 \\
\hline Aldosterone antagonist & $399 / 928(43.0 \%)$ & $189 / 451(41.9 \%)$ & $210 / 477(44.0 \%)$ & .515 \\
\hline Antiarrhythmic therapy & $132 / 927(14.2 \%)$ & $66 / 451(14.6 \%)$ & $66 / 476(13.9 \%)$ & .738 \\
\hline Digoxin & $432 / 928(46.6 \%)$ & $219 / 451(48.6 \%)$ & $213 / 477(44.7 \%)$ & .233 \\
\hline ICD at baseline & $411 / 928(44.3 \%)$ & $193 / 451(42.8 \%)$ & $218 / 477(45.7 \%)$ & .373 \\
\hline
\end{tabular}

Values are median (interquartile range) or n (\%). Abbreviations: $M I$, Myocardial infarction; $M R$, mitral regurgitation; $B$, baseline; $3 M, 3$ months; $A C E$, angiotensin-converting enzyme; $A R B$, angiotensin receptor blocker; $I C D$, implantable cardiac defibrillator. 


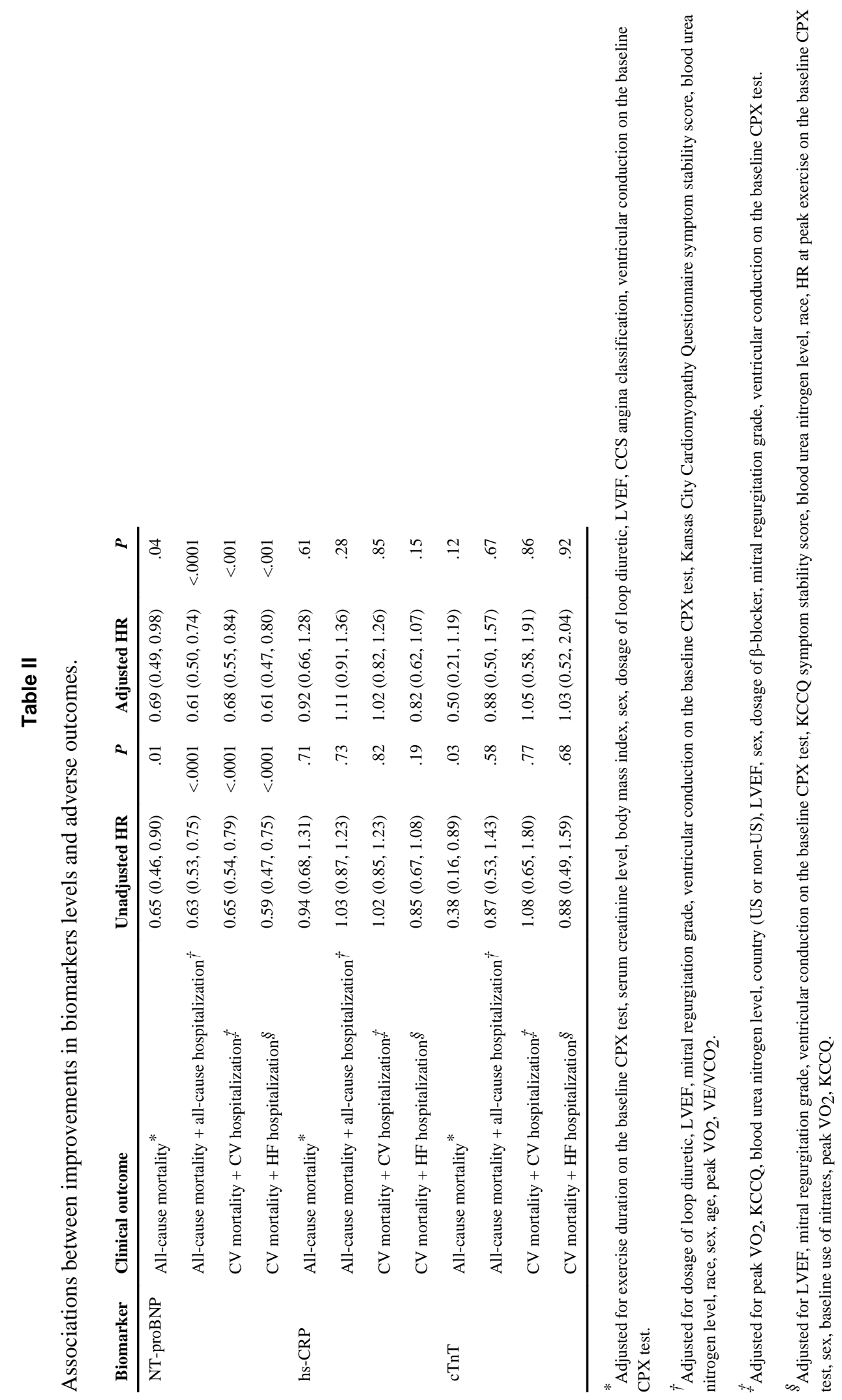

